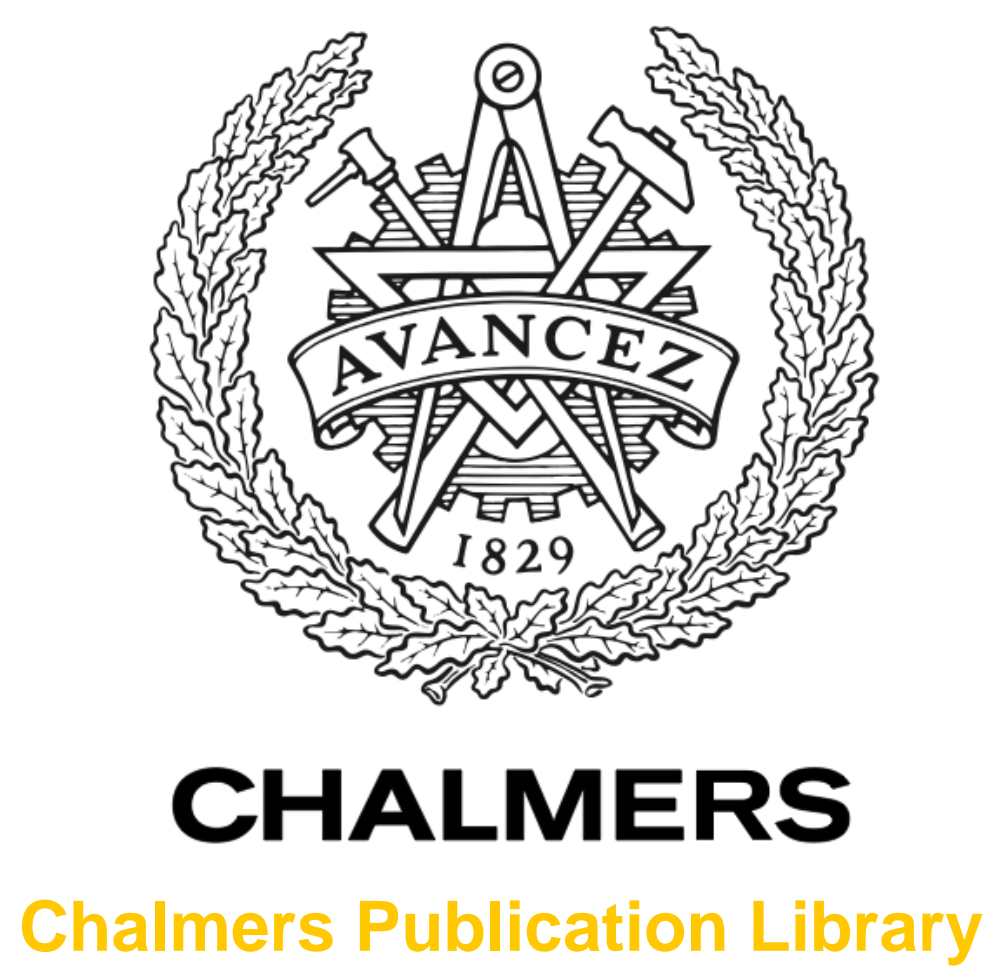

\title{
Detection of Forest Change and Robust Estimation of Forest Height from Two-Level Model Inversion of Multi-Temporal Single-Pass InSAR Data
}

This document has been downloaded from Chalmers Publication Library (CPL). It is the author's version of a work that was accepted for publication in:

\section{IEEE International Geoscience and Remote Sensing Symposium}

Citation for the published paper:

Soja, M. ; Persson, H. ; Ulander, L. (2015) "Detection of Forest Change and Robust

Estimation of Forest Height from Two-Level Model Inversion of Multi-Temporal Single-

Pass InSAR Data". IEEE International Geoscience and Remote Sensing Symposium

Downloaded from: http://publications.lib.chalmers.se/publication/226249

Notice: Changes introduced as a result of publishing processes such as copy-editing and formatting may not be reflected in this document. For a definitive version of this work, please refer to the published source. Please note that access to the published version might require a subscription. 


\title{
DETECTION OF FOREST CHANGE AND ROBUST ESTIMATION OF FOREST HEIGHT FROM TWO-LEVEL MODEL INVERSION OF MULTI-TEMPORAL, SINGLE-PASS INSAR DATA
}

\author{
Maciej J. Soja ${ }^{1)}$, Henrik J. Persson ${ }^{2)}$, and Lars M. H. Ulander ${ }^{1)}$ \\ ${ }^{1)}$ Chalmers University of Technology, Gothenburg, Sweden \\ ${ }^{2}$ Swedish University of Agricultural Sciences, Umeå, Sweden
}

\begin{abstract}
In this paper, forest change detection and forest height estimation are studied using two-level model (TLM) inversion of multi-temporal TanDEM-X (TDM) data. Parameter $\Delta h$, describing the distance between ground and vegetation levels, is kept constant for all acquisitions, whereas parameter $\mu$, the area-weighted backscatter ratio, changes with acquisition. Two multi-temporal sets of TDM data, acquired over the hemi-boreal test site Remningstorp, situated in southern Sweden, are studied: one consisting of 12 acquisitions made in the summers of 2011, 2012, 2013, and 2014 with heights-ofambiguity (HOAs) between $32 \mathrm{~m}$ and $63 \mathrm{~m}$, and one consisting of 33 acquisitions made between August 2013 and August 2014 with HOAs between $38 \mathrm{~m}$ and $195 \mathrm{~m}$. The first dataset is used to show that commercial thinnings and clearcuts can be detected by studying the canopy density estimate $\eta_{0}=1 /(1+\mu)$. The second dataset is used to show that seasonal change can be observed in $\eta_{0}$ for deciduous plots, but not for coniferous plots. Moreover, it is shown that $1.3 \Delta h$ is a good estimate of the basal area-weighted (Lorey's) height, with a correlation coefficient equal to 0.98 and a root-meansquare error of $0.9 \mathrm{~m}$.
\end{abstract}

Index Terms - canopy density, forest height, clear-cut, two-level model (TLM), TanDEM-X

\section{INTRODUCTION}

Accurate mapping of forest change is needed for applications such as commercial forestry, disaster monitoring, and detection of unlawful deforestation. Synthetic-aperture radar is an active microwave sensor, meaning that it has weather- and sunlight-independent capabilities. Moreover, the resolution of modern spaceborne SAR systems can be of the order of meters, which is advantageous for resolving features on the scale of a few trees.

The TanDEM-X (TDM) system from the German Aerospace Center (DLR) consists of two, almost identical-twin satellites TerraSAR-X (launched in June 2007) and TanDEM-X (launched in June 2010), designed to operate in a tight formation flight in a helical orbit. During the main operational mode, the two satellites were acquiring global data with spatial baselines of a few hundreds of meters and negligible temporal baselines, which made it possible to use the principles of single-pass interferometric SAR (InSAR) to create the first, high-resolution, fully-global digital elevation model (DEM). With an external high-resolution digital terrain model (DTM), TDM data can also be used to measure the elevation of the scattering phase center above ground, which is related to the forest height, canopy density, and biomass $[1,2,3,4,5]$.

In [4], a two-level model (TLM) was introduced, and it was shown that direct inversion of the TLM can be used to estimate forest height and canopy density from single-polarized TDM data, with root-mean square errors better than $10 \%$, for heights-of-ambiguity around 50-60 meters and without any training data. In [5], a regression model was used to link the inverted TLM parameters to forest biomass, and it was shown that biomass estimation can be done from single-polarized TDM data with root-mean square errors of the order of $15 \%$, with only limited local model training required.

In this paper, the TLM will be fitted to multi-temporal TDM data, and the estimated TLM parameters will be studied for their potential to detect forest change caused by harvesting and seasonal variations. Forest height estimation will also be evaluated. TDM data acquired over the hemi-boreal, Swedish test site Remningstorp will be used.

\section{TWO-LEVEL MODEL (TLM)}

In the two-level model (TLM) [4, 5], forest is represented by two discrete scattering levels, ground and vegetation, the latter with gaps. The vertical separation of the levels is denoted by $\Delta h$ and the relative contribution of each level to the total backscatter is controlled by parameter

$$
\mu=\frac{\sigma_{\mathrm{gr}}^{0}}{\sigma_{\mathrm{veg}}^{0}} \frac{1-\eta}{\eta}
$$

where $\sigma_{\mathrm{gr}}^{0}$ and $\sigma_{\mathrm{veg}}^{0}$ are the effective scattering coefficients for the ground and vegetation levels, respectively, and $\eta$ is the area-fill factor, defined as the fraction of the total area covered

(C) 2015 IEEE. Personal use of this material is permitted. Permission from IEEE must be obtained for all other uses, in any current or future media, including reprinting/republishing this material for advertising or promotional purposes, creating new collective works, for resale or redistribution to servers or lists, or reuse of any copyrighted component of this work in other works. 


\section{Unchanged plots}

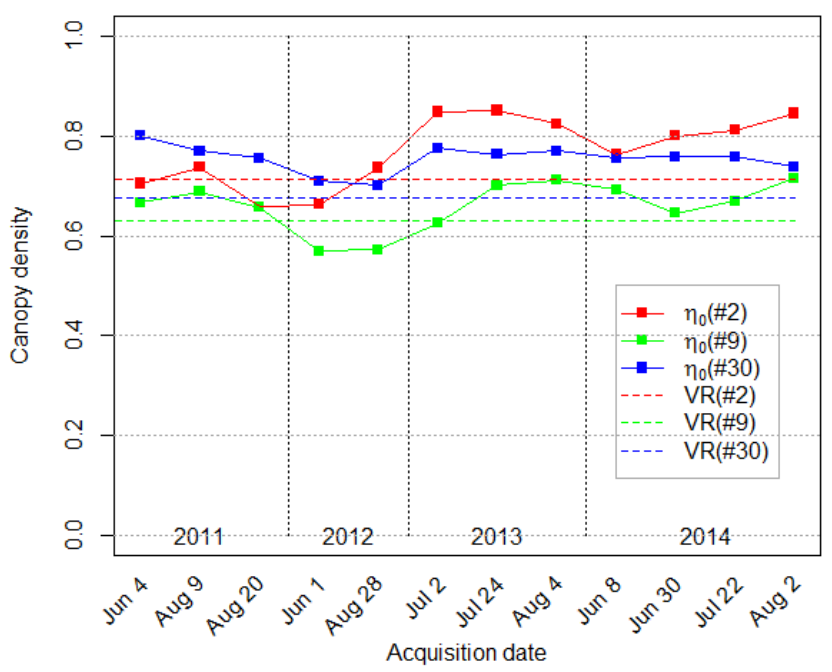

Harvested plots

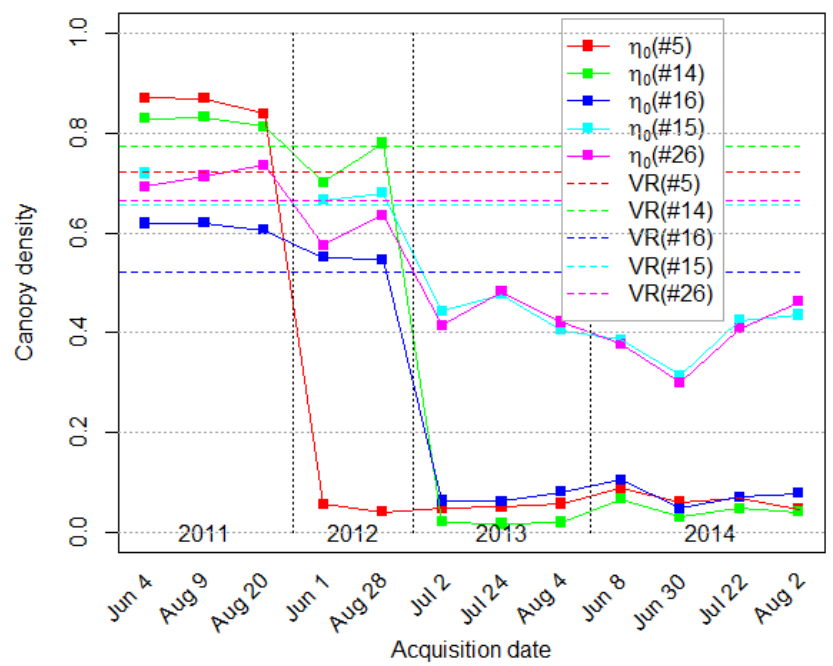

Fig. 1. Temporal change of canopy density estimate $\eta_{0}$ between the summers of 2011 and 2014 for two groups of plots: unchanged plots and harvested plots (clear-cuts: \#5, \#14, \#16; commercial thinnings: \#15, \#26). VR (vegetation ratio) is a lidar-based canopy density estimate, measured in 2010.

by the vegetation level; $\eta$ is frequently used in studies treating the interferometric water cloud model (IWCM) [2].

The TLM models the ground-corrected complex correlation coefficient $\tilde{\gamma}_{\mathrm{gc}}$ as:

$$
\tilde{\gamma}_{g c}=\frac{\mu+\exp \left(i 2 \pi \frac{\Delta h}{\mathrm{HOA}}\right)}{\mu+1}
$$

where HOA is the height-of-ambiguity, i.e., the vertical height difference giving a $2 \pi$-phase shift of the interferogram. For a bistatic acquisition scenario, $\mathrm{HOA}=\lambda R \sin \theta / B_{\perp}$, where $\lambda$ is the wavelength, $R$ is the average range, $\theta$ is the average angle of incidence, and $B_{\perp}$ is the perpendicular baseline. Note that in this text, the term "ground-corrected" refers to the fact that the terrain phase has been removed during interferogram creation.

In [4], expression (2) was fitted to a single, groundcorrected complex correlation coefficient, and it was shown that the level distance $\Delta h$ and the uncorrected area-fill factor $\eta_{0}=1 /(1+\mu)$ can be used as estimates of forest height and canopy density, respectively, the first correlated with lidar height $\mathrm{H} 50$ (50th percentile of lidar returns above $1 \mathrm{~m}$ or $10 \%$ of the maximal height) and the second correlated with lidar vegetation ratio (VR, fraction of the number of lidar returns above $1 \mathrm{~m}$ or $10 \%$ of the maximal height to all lidar returns).

Following the observations from [4], it can be concluded that $\Delta h$ represents an effective height at which a majority of the canopy scatterers are located, and it is thus related to the vertical structure of the forest. For a homogeneous $\mathrm{plot} / \mathrm{stand}$, it is therefore reasonable to assume that $\Delta h$ is temporally more stable than $\mu ; \Delta h$ should primarily be af- fected by growth, clear-cutting, and natural disasters (which change the vertical structure), whereas $\mu$ should primarily be affected by the more limited management procedures such as thinnings, as well as seasonal changes (which are assumed to change the canopy density more than the vertical structure of a homogeneous plot/stand).

In this paper, expression (2) will be fitted to multitemporal, multi-baseline TanDEM-X data consisting of $N$ single-polarized, ground-corrected complex correlation coefficients. For better stability, and following the reasoning from the previous paragraph, $\Delta h$ will be assumed constant in time, whereas $\mu$ will be allowed to change between acquisitions. This will result in the over-determined system of $2 \mathrm{~N}$ equations with $N+1$ unknowns, which will be solved using non-linear least squares.

\section{EXPERIMENTAL DATA}

In this study, the hemi-boreal test site Remningstorp, situated in southern Sweden, is used. Within Remningstorp, there are 32, 0.5-hectare circular forest plots. Extensive in situ and lidar data were collected in 2010, 2011, and 2014. Some of the plots underwent management activities between 2010 and 2014. The state of the plots at each TDM acquisition was assessed qualitatively using information about forest management procedures provided by the manager-on-site, analysis of optical SPOT-5 imagery, and field visits. Three plots were fully harvested (one in early 2012 and two in early 2013), and in 2013 additional two plots underwent commercial thinning (roughly $60 \%$ of biomass was harvested). 


\section{Coniferous plots}

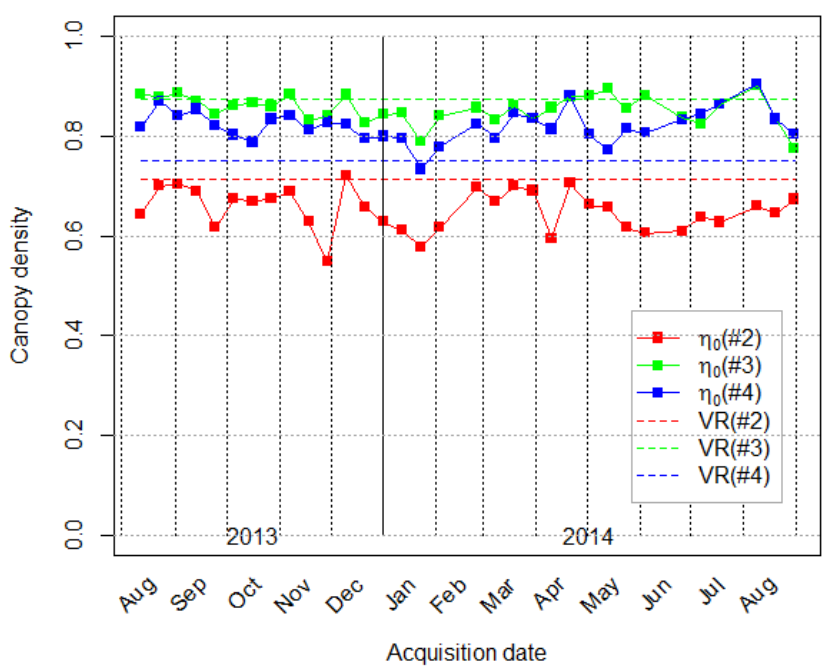

Deciduous plots

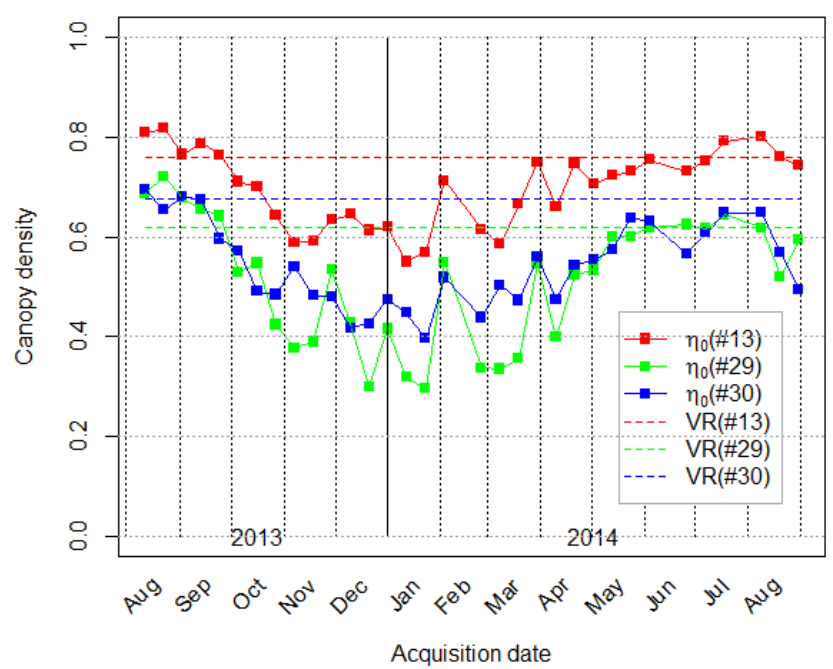

Fig. 2. Temporal change of canopy density estimate $\eta_{0}$ between August 2013 and August 2014 for two groups of plots: coniferous plots and plots with significant deciduous contribution to the total biomass (at least 40\%). VR (vegetation ratio) is a lidar-based canopy density estimate, measured in 2010.

Two multi-temporal sets of TanDEM-X data acquired between 2011 and 2014 are used. The first set consists of $12 \mathrm{VV}$ polarized acquisitions made in the summers of 2011, 2012, 2013, and 2014, with HOAs in the interval $32 \mathrm{~m}-63 \mathrm{~m}$ (median: $54 \mathrm{~m})$ and $\theta \approx 41^{\circ}$. A subset of these data, spanning years 2011-2013, has previously been used in [4, 5]. The second data set consists of $33 \mathrm{VV}$-polarized acquisitions made between August 2013 and August 2014, with HOAs in the interval $38 \mathrm{~m}-195 \mathrm{~m}$ (median: $51 \mathrm{~m}$ ) and $\theta \approx 34^{\circ}$.

All TDM data have been interferometrically processed using the national, $2 \mathrm{~m} \times 2 \mathrm{~m}$ digital terrain model (DTM) and the same methods as presented in $[2,4,5]$, with the exception that a coherence calibration step has been added for the current paper. During coherence calibration, the obtained ground-corrected correlation coefficient was compensated for SNR and system decorrelation by division with a calibration factor. For simplicity and based on observations in data, the calibration factor was chosen to be constant and equal to 0.95 for all plots and acquisitions.

\section{RESULTS}

In Fig. 1, temporal change of $\eta_{0}$ is shown for the first data set, spanning four consecutive summers. Eight plots are studied in total: three representative unchanged plots, three plots which have been clear-cut at some point during the study (for these plots, the loss of tree biomass was 100\%), and two plots which have been thinned at some point during the study (for these plots, the loss of tree biomass was estimated to approximately $60 \%$ ). As it can be observed, harvesting activities cause the canopy density estimate $\eta_{0}$ to decrease, to approximately 0.1 for clear-cuts and 0.4 for thinnings. It can also be observed that $\eta_{0}$ shows relatively good agreement with the lidar-based estimate of canopy density VR, i.e., the vegetation ratio measured as the fraction of all lidar returns coming from above $1 \mathrm{~m}$ or $10 \%$ of the maximal return.

In Fig. 2, temporal change of $\eta_{0}$ is shown for the second data set, spanning roughly one year between August 2013 and August 2014. Six plots are studied in total: three plots dominated by coniferous trees (spruce and pine, contributing to more than $98 \%$ of biomass), and three plots with the highest contribution of deciduous trees (primarily birch, contributing to $40 \%$ or more of the total biomass). The leaf-off effect can be observed for the deciduous plots as a significant decrease of the canopy density estimate $\eta_{0}$. It can also be observed that $\eta_{0}$ shows relatively good agreement with VR during the summer months.

In Fig. 3, height estimation results are shown for the two data sets, in a comparison with the basal area-weighted (Lorey's) height $H$. A very good correlation is observed $(r \approx 0.98$ and $r \approx 0.95$ for the first and second data sets, respectively). Moreover, it is observed that a multiplication of $\Delta h$ with 1.3 gives a very good estimate of $H$, with the respective root-mean-square errors around $0.95 \mathrm{~m}$ and $1.90 \mathrm{~m}$, or roughly $4 \%$ and $8 \%$ of the mean $H$.

\section{SUMMARY AND DISCUSSION}

This paper proposes a multi-temporal two-level model inversion procedure and it shows using TanDEM-X data that the 
Summers 2011-2014

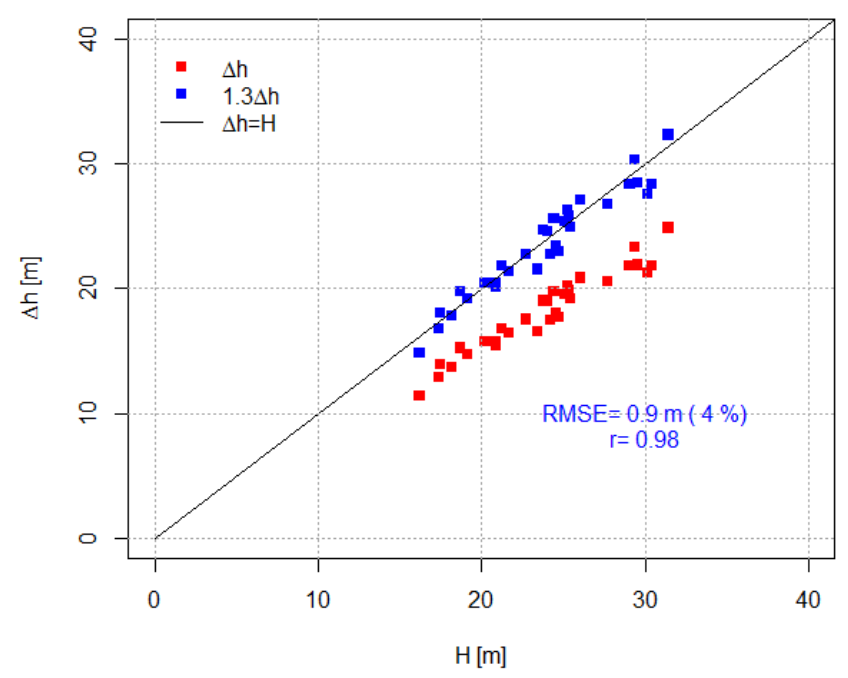

August 2013-August 2014

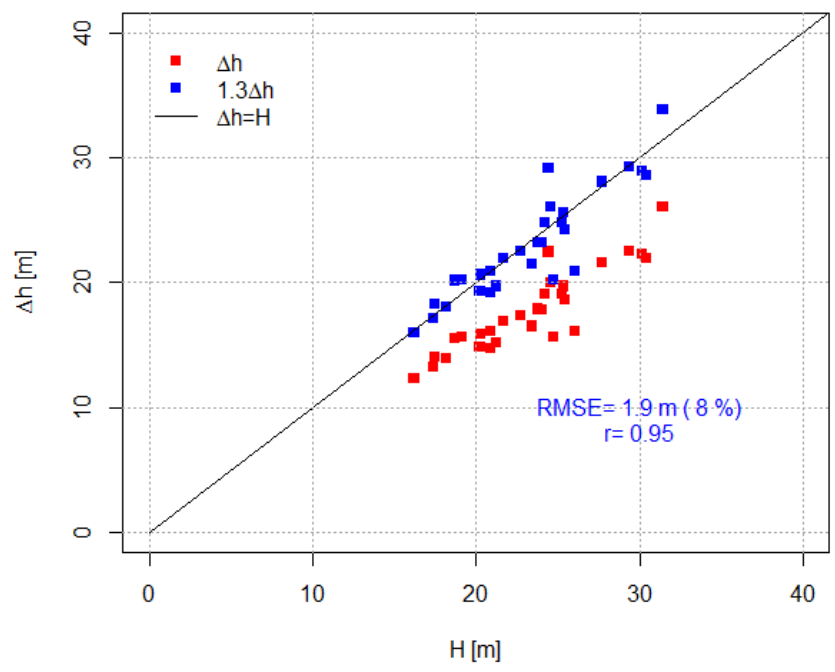

Fig. 3. Scatter plots with height estimation results from multi-temporal TLM inversion. $H$ is the basal area-weighted (Lorey's) height, estimated from field inventories conducted in 2010-2011. In the first time series (summers 2011-2014), all 32 plots are included. In the second time series (August 2013-August 2014), the three clear-cuts have been excluded.

inversion provides useful parameters. The proposed inversion procedure assumes $\Delta h$ constant for all acquisitions, which implies that the vertical structure is unaffected by short-term seasonal and man-made changes, whereas $\mu$ is allowed to change between acquisitions. The estimated $\Delta h$ is found highly correlated with forest height, and a multiplication with 1.3 gives a very good estimate of forest height. A time series study of the canopy density estimate $\eta_{0}=1 /(1+\mu)$ shows that large harvesting activities, such as commercial thinning and clear-cutting, as well as seasonal changes, can be detected.

The remaining variations of $\eta_{0}$ in Figs. 1-2, which cannot easily be explained by human-induced or seasonal changes, are probably caused by modeling inaccuracies, although actual variations in canopy density may also contribute. The influence of weather has not been studied here, but it is an important factor and should be included in the future. Also, growth has not been modelled, which may be the main cause of the slightly worse height estimation results obtained for the second data set in Fig. 3. Further work also includes using multi-temporal TLM inversion for biomass change mapping from a time series of TDM images.

\section{ACKNOWLEDGMENTS}

The authors would like to thank DLR for the TDM data, Swedish Land Survey for the DTM data, ESA for the BioSAR 2010 lidar data, as well as the Swedish National Space Board and the European Union's Seventh Framework Programme (Advanced SAR) for the funding of this work.

\section{REFERENCES}

[1] Svein Solberg, Rasmus Astrup, Johannes Breidenbach, Barbi Nilsen, and Dan Weydahl, "Monitoring spruce volume and biomass with InSAR data from TanDEMX," Remote Sensing of Environment, vol. 139, pp. 60-67, 2013.

[2] Jan I. H. Askne, Johan E. S. Fransson, Maruzio Santoro, Maciej J. Soja, and Lars M. H. Ulander, "Model-based biomass estimation of a hemi-boreal forest from multitemporal TanDEM-X acquisitions," Remote Sensing, vol. 5, pp. 5574-5597, 2013.

[3] Florian Kugler, Daniel Schulze, Irena Hajnsek, Hans Pretzsch, and Kostantinos P. Papathanassiou, "TanDEMX Pol-InSAR performance for forest height estimation," IEEE Transactions on Geoscience and Remote Sensing, vol. 52, no. 10, pp. 6404-6422, 2014.

[4] Maciej Jerzy Soja, Henrik Persson, and Lars M. H. Ulander, "Estimation of forest height and canopy density from a single InSAR correlation coefficient," Geoscience and Remote Sensing Letters, vol. 12, no. 3, pp. 646-650, 2015.

[5] Maciej Jerzy Soja, Henrik J. Persson, and Lars M. H. Ulander, "Estimation of forest biomass from two-level model inversion of single-pass InSAR data," IEEE Transactions on Geoscience and Remote Sensing, 2015, (published on-line). 